\title{
Two-Fluid Mixed Magnetoconvection Flow in a Vertical Enclosure
}

\author{
J. Prathap Kumar ${ }^{1}$, J.C. Umavathi ${ }^{1 \dagger}$ and B.M. Biradar ${ }^{2}$ \\ ${ }^{I}$ Department of Mathematics, Gulbarga University, Gulbarga-585 106, Karnataka, India \\ ${ }^{2}$ Department of Mathematics, Rural Engineering College, Bhalki-585 328, Karnataka, India \\ †Corresponding Author Email: jc_uma11@yahoo.com
}

(Received February 28, 2010; accepted July 12, 2011)

\begin{abstract}
The problem of steady, laminar flow and heat transfer of an electrically conducting fluid through vertical channel in the presence of uniform transverse magnetic field is formulated using a two-fluid continuum model. Combined free and forced convection inside the channel is considered. The effects of viscous and ohmic dissipations are included in the energy equation. Both walls are kept either at the same or different temperatures such as isoflux-isothermal and isothermal-isoflux conditions. Governing equations in cartesian co-ordinates are solved analytically using regular perturbation technique to develop the expression for velocity and temperature. Velocity, temperature and Nusselt number are presented graphically. Effects of pertinent parameters, such as Hartmann number, electric field load parameter, viscosity ratio, width ratio and conductivity ratio are determined.
\end{abstract}

Keywords: Two-fluid, Magnetoconvection, Vertical enclosure, Isoflux-isothermal.

\section{NOMENCLATURE}
A
constant defined in Eq. (8)
$h_{1} \quad$ width of the region-I
$b$ thermal expansion coefficient ratio $\left(\frac{\beta_{2}}{\beta_{1}}\right)$
$h_{2} \quad$ width of the region-II
$B_{0} \quad$ magnetic field
$K \quad$ ratio of the thermal conductivities $\left(\frac{K_{1}}{K_{2}}\right)$
$B r \quad$ Brinkman number $\left(\frac{\mu_{1} U_{0}^{(1)^{2}}}{K_{1} \Delta T}\right)$
$K_{1} \quad$ thermal conductivity of the fluid in region-I
$C_{p} \quad$ specific heat at constant pressure
$E_{0} \quad$ applied electric field
$E \quad$ electric field load parameter $\left(\frac{E_{0}}{B_{0} U_{0}^{(1)}}\right)$
g acceleration due to gravity
$K_{2}$ thermal conductivity of the fluid in region-II
Gr Grashof number $\left(\frac{g \beta_{1} h_{1}^{3} \Delta T}{v_{1}^{2}}\right)$
$G R \quad$ mixed convection parameter $\left(\frac{G r}{\mathrm{Re}}\right)$
M Hartmann number $\left(h_{1} B_{0} \sqrt{\frac{\sigma_{e}}{\mu_{1}}}\right)$
$m \quad$ ratio of the viscosities $\left(\frac{\mu_{1}}{\mu_{2}}\right)$
$n \quad$ ratio of the densities $\left(\frac{\rho_{2}}{\rho_{1}}\right)$
$p \quad$ dimensional pressure
$\operatorname{Re} \quad$ Reynolds number $\left(\frac{U_{0}^{(1)} h_{1}}{v_{1}}\right)$
defined in Eq. (23)
$h \quad$ width ratio $\left(\frac{h_{2}}{h_{1}}\right)$
$T$ temperature
$U_{0}^{(i)} \quad$ reference velocity $\left(-\frac{d P}{d X} \frac{h_{i}^{2}}{48 \mu_{i}}\right)$
$\bar{u}_{i} \quad$ average velocity
$T_{1}, T_{2} \quad$ temperature of the boundaries
$\mathrm{X}, \mathrm{Y}$ space co-ordinates
$u \quad$ velocity
Greek symbols
$\beta \quad$ coefficient of thermal expansion
$v \quad$ kinematic viscosity 


$\begin{array}{ll}\alpha & \text { thermal diffusivity } \\ \sigma_{e} & \text { electrical conductivity } \\ \mu & \text { viscosity } \\ \rho & \text { density of the fluid }\end{array}$

\section{INTRODUCTION}

Mixed convection is defined as heat transfer situations where both natural convection and forced convection heat transfer mechanisms interact. In a vertical passage, the internal main flow can be either upward or downward. The upward forced flow is termed 'assisted' flow because the natural convection created by buoyancy is in the same direction as the bulk flow. In contrast, the downward flow is called 'opposed' flow based on its direction opposite to the natural convection. In the past twenty years, mixed convection in a vertical heated channel has received considerable attention due to its extensive practical applications, including turbine rotor blade internal cooling systems, cooling of nuclear reactors and electronic components. Tao (1960) analyzed laminar fully developed mixed convection flow in a vertical parallel-plate channel with uniform wall temperatures. Aung and Worku (1986a, $1986 \mathrm{~b}$ ) discussed the theory of combined free and forced convection in a vertical channel with flow reversal conditions for both developing and fully developed flows. Aung and Worku (1986b) assumed that the walls of the channel were having asymmetric temperatures. The case of developing mixed convection flow in ducts with asymmetric wall heat fluxes was analyzed by the same authors (Aung and Worku, 1987). A comprehensive review of the literature dealing with mixed convection in internal flow was reported by Aung (1987). Cheng et al. (1990), Hamadah and Wirtz (1991) and Ingham et al. (1988) also reported on, flow reversal situation in mixed convection in a vertical channel for different wall heating conditions.

The problem concerning the flow of immiscible fluids has a definite role in chemical engineering and in medicine. In view of this, Bird et al. (1960) obtained an exact solution for the laminar flow of two immiscible fluids between two parallel plates. Mitra (1982) analyzed the unsteady flow of two electrically conducting fluids between two rigid parallel plates.

Since the last three decades research in nuclear power safety has motivated the study of the buoyancy-driven convection in fluids with volumetric heat generation. Recently, the problem of convective heat transfer in a layered system attracted attention due to possible core melt stratification in a postulated severe accident scenario in a light water reactor (Sehgal, 2001). Besides the reactor safety research, thermal convection in a double-layer system is of interest in chemical engineering and geophysics. Much research effort has been directed at the problem of natural convection in a uniform pool with internal heat generation (Dhir, 1997). However, only a few studies have focused on the subject of thermal convection in volumetrically heated stratified layers. Fieg (1976) investigated the natural convection characteristics of two stratified immiscible liquid layers with internally heated lower layer. The $\varepsilon \quad$ dimensionless parameter defined in Eq.(42)

$\Delta T \quad$ difference in temperature $\left(T_{2}-T_{1}\right)$

$\theta_{i} \quad$ nondimensional temperature $\frac{\left(T_{i}-T_{0}\right)}{\Delta T}$

temperature was maintained equal at the top and bottom boundaries. Heptane and water were used as lighter and heavier liquids, respectively. The important conclusion was that the two layers behaved as if separated by a rigid highly conductive wall. Schramm and Reineke (1978) studied experimentally and numerically the natural convection in a rectangular channel filled with two immiscible fluids of different physical properties. Kulacki and Nguen (1981) studied hydrodynamic instability and thermal convection in a horizontal layer of two immiscible fluids with internal heat generation in the lower layer.

There has been some theoretical and experimental work on the stratified laminar flow of two immiscible liquids in a horizontal pipe (see, Charles and Lilleleht, 1965, Bentwich, 1964, and Pacham and Shail, 1971). The interest in this configuration stems from the possibility of reducing the power required to pump oil in a pipeline by the suitable addition of water. Shail (1973) investigated theoretically the possibility of using a twophase system to obtain increased flow rates in an electromagnetic pump. Hartmann flow of a conducting fluid and non-conducting fluid layer contained in a channel has been studied by Shail (1973). His results predicted that an increase of the order $30 \%$ can be achieved in the flow rate for suitable ratios heights and viscosities of the fluids. Malashetty and Leela (1991, 1992) reported closed-form solutions for the two-phase flow and heat transfer situation in a horizontal channel for which both phases are electrically conducting. Malashetty and Umavathi (1997) studied two-phase MHD flow and heat transfer in an inclined channel in the presence of buoyancy effects for the situation where only one of the phases is electrically conducting. Malashetty et al. $(2000,2001)$ analyzed the problem of fully developed two fluid magnetohydrodynamic flows with and without applied electric field in an inclined channel. Recently, Malashetty et al. (2006) studied the magneto convection in a vertical channel.

Much work is not found in the literature in the presence of electromagnetic field. Keeping in view numerous applications of two-immiscible fluids, it is the objective of the paper to investigate theoretically, mixed convection of electrically conducting and electrically non-conducting immiscible fluids in a vertical channel.

\section{Mathematical Formulation}

The geometry under consideration illustrated in Fig. 1 consists of two infinite parallel plates maintained at different or equal constant temperatures extending in the $\mathrm{X}$ and $\mathrm{Z}$ directions. The region $-\frac{h_{1}}{2} \leq Y \leq 0$ is occupied by a viscous, electrically conducting fluid of density $\rho_{1}$, viscosity $\mu_{1}$, thermal conductivity $K_{1}$, thermal expansion coefficient $\beta_{1}$ and electrical conductivity $\sigma_{e}$. 


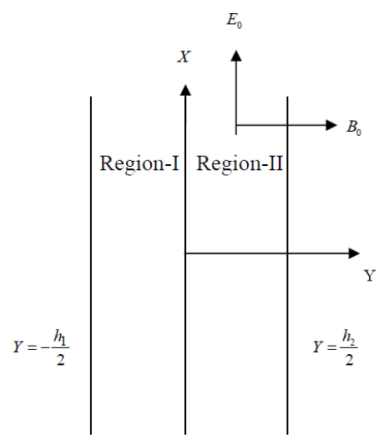

Fig. 1. Physical configuration.

The region $0 \leq Y \leq \frac{h_{2}}{2}$ is occupied by another viscous, non-conducting fluid of density $\rho_{2}$, viscosity $\mu_{2}$, thermal conductivity $K_{2}$, and thermal expansion coefficient $\beta_{2}$. A uniform magnetic field $B_{0}$ is applied normal to the plates and the uniform electric field $E_{0}$ is applied across the channel. The fluids are assumed to have constant properties except the density in the buoyancy term in momentum equation. A fluid rises in the channel driven by buoyancy forces. The transport properties of both the fluids are assumed to be constant. The fluids in both the regions are considered to be incompressible and the flow is steady, laminar and fully developed. It is assumed that the only non-zero component of the velocity $\vec{q}$ is the $X$-component $U_{i}(i=1,2)$. Thus, as a consequence of the mass balance equation, one obtains

$$
\frac{\partial U_{i}}{\partial X}=0
$$

so that $U_{i}$ depends only on $\mathrm{Y}$.

The stream wise and the transverse momentum balance equations yields (Arpaci and Larsen, 1984).

Region-I

$g \beta_{1}\left(T_{1}-T_{0}\right)-\frac{1}{\rho_{1}} \frac{\partial P}{\partial X}+v_{1} \frac{d^{2} U_{1}}{d Y^{2}}-\frac{\sigma_{e}}{\rho_{1}}\left(E_{0}+B_{0} U_{1}\right) B_{0}=0$

The $\mathrm{Y}$-momentum balance equation can be expressed as

$\frac{\partial P}{\partial Y}=0$

Region-II

$g \beta_{2}\left(T_{2}-T_{0}\right)-\frac{1}{\rho_{2}} \frac{\partial P}{\partial X}+v_{2} \frac{d^{2} U_{2}}{d Y^{2}}=0$

$\frac{\partial P}{\partial Y}=0$

where $P=p+\rho_{0} g x\left(\right.$ for $\left.P_{1}=P_{2}=P\right)$ is the difference between the pressure and the hydrostatic pressure. On account of Eqs. (3) and (5), $P$ depends only on $X$ so that Eqs. (2) and (4) can be rewritten as

Region- $I$ $g \beta_{1}\left(T_{1}-T_{0}\right)-\frac{1}{\rho_{1}} \frac{d P}{d X}+v_{1} \frac{d^{2} U_{1}}{d Y^{2}}-\frac{\sigma_{e}}{\rho_{1}}\left(E_{0}+B_{0} U_{1}\right) B_{0}=0$

Region-II

$g \beta_{2}\left(T_{2}-T_{0}\right)-\frac{1}{\rho_{2}} \frac{d P}{d X}+v_{2} \frac{d^{2} U_{2}}{d Y^{2}}=0$

Let us assume that the walls of the channel are isothermal. In particular, the temperature of the boundary at $Y=-\frac{h_{1}}{2}$ is $T_{1}$, while the temperature at $Y=\frac{h_{2}}{2}$ is $T_{2}$, with $T_{2} \geq T_{1}$. These boundary conditions are compatible with Eqs. (6) and (7) if and only if $\frac{d P}{d X}$ is independent of $X$. Therefore, there exists a constant $A$ such that

$\frac{d P}{d X}=A$

On account of Eq. (8) and by evaluating the derivatives of Eqs. (6) and (7) with respect to $X$, one obtains

$\frac{d T_{1}}{d X}=0$
$\frac{d T_{2}}{d X}=0$

so that the temperature also depends only on $Y$. By taking into account the effect of viscous dissipation, the energy balance equation can be written as

Region-I

$\alpha_{1} \frac{d^{2} T_{1}}{d Y^{2}}+\frac{v_{1}}{C_{p}}\left(\frac{d U_{1}}{d Y}\right)^{2}+\frac{\sigma_{e}}{\rho_{1} C_{p}}\left(E_{0}+B_{0} U_{1}\right)^{2}=0$

Region-II

$\alpha_{2} \frac{d^{2} T_{2}}{d Y^{2}}+\frac{v_{2}}{C_{p}}\left(\frac{d U_{2}}{d Y}\right)^{2}=0$

Equations (6), (11), (7) and (12) allow one to obtain differential equation for $U_{i}$ namely

Region-I

$\frac{d^{4} U_{1}}{d Y^{4}}-\frac{\sigma_{e} B_{0}^{2}}{\mu_{1}} \frac{d^{2} U_{1}}{d Y^{2}}=\frac{\rho_{1} g \beta_{1}}{K_{1}}\left(\frac{d U_{1}}{d Y}\right)^{2}+\frac{g \beta_{1} \sigma_{e}}{K_{1} V_{1}}\left(E_{0}+B_{0} U_{1}\right)^{2}$

Region-II

$\frac{d^{4} U_{2}}{d Y^{4}}=\frac{\rho_{2} g \beta_{2}}{K_{2}}\left(\frac{d U_{2}}{d Y}\right)^{2}$

The boundary conditions on $U_{i}$ are both no-slip conditions

$\begin{array}{ll}U_{1}=0 & \text { at } Y=-\frac{h_{1}}{2} \\ U_{2}=0 & \text { at } Y=\frac{h_{2}}{2}\end{array}$ 
and those induced by the boundary conditions on $T$ and by Eqs. (6) and (7) are

$U_{1}(0)=U_{2}(0)$

$\frac{d^{2} U_{1}}{d Y^{2}}=\frac{A}{\mu_{1}}+\frac{\sigma_{e} E_{0} B_{0}}{\mu_{1}}+\frac{g \beta_{1} \Delta T R_{T}}{2 v_{1}}$ at $Y=-\frac{h_{1}}{2}$

$\frac{d^{2} U_{2}}{d Y^{2}}=\frac{A}{\mu_{2}}-\frac{g \beta_{2} \Delta T R_{T}}{2 v_{2}} \quad$ at $Y=\frac{h_{2}}{2}$

$\mu_{1} \frac{d U_{1}}{d Y}=\mu_{2} \frac{d U_{2}}{d Y} \quad$ at $Y=0$

$\frac{d^{2} U_{1}}{d Y^{2}}-\frac{\sigma_{e}}{\mu_{1}}\left(E_{0}+B_{0} U_{1}\right) B_{0}=\frac{\mu_{2}}{\mu_{1}} \frac{\rho_{1}}{\rho_{2}} \frac{\beta_{1}}{\beta_{2}} \frac{d^{2} U_{2}}{d Y^{2}}+\frac{A}{\mu_{1}}\left(1-\frac{\rho_{1}}{\rho_{2}} \frac{\beta_{1}}{\beta_{2}}\right)$

$\frac{d^{3} U_{1}}{d Y^{3}}-\frac{\sigma_{e} B_{0}^{2}}{\mu_{1}} \frac{d U_{1}}{d Y}=\frac{\mu_{2}}{\mu_{1}} \frac{\rho_{1}}{\rho_{2}} \frac{K_{2}}{K_{1}} \frac{\beta_{1}}{\beta_{2}} \frac{d^{3} U_{2}}{d Y^{3}}$

at $Y=0$

The Eqs. (13) to (22) can be written in the dimensionless form by employing the dimensionless quantities

$$
\begin{aligned}
& u_{1}=\frac{U_{1}}{U_{0}^{(1)}} ; u_{2}=\frac{U_{2}}{U_{0}^{(2)}} ; \theta_{1}=\frac{T_{1}-T_{0}}{\Delta T} ; \theta_{2}=\frac{T_{2}-T_{0}}{\Delta T} ; y_{1}=\frac{Y_{1}}{h_{1}} ; y_{2}=\frac{Y_{2}}{h_{2}} \\
& G r=\frac{g \beta \Delta T h_{1}^{3}}{v_{1}^{2}} ; \operatorname{Re}=\frac{U_{0}^{(1)} h_{1}}{v_{1}} ; B r=\frac{\mu_{1} U_{0}^{(1)^{2}}}{K_{1} \Delta T} ; G R=\frac{G r}{\operatorname{Re}} ; R_{T}=\frac{T_{2}-T_{1}}{\Delta T} \\
& M^{2}=\frac{\sigma_{e} B_{0}^{2} h_{1}^{2}}{\mu_{1}} ; E=\frac{E_{0}}{B_{0} U_{0}^{(1)}} ;
\end{aligned}
$$

The reference velocity $U_{0}^{(i)}$ and the reference temperature $T_{0}$ are given by

$$
U_{0}^{(1)}=-\frac{A h_{1}^{2}}{48 \mu_{1}} ; U_{0}^{(2)}=-\frac{A h_{2}^{2}}{48 \mu_{2}} ; T_{0}=\frac{T_{1}+T_{2}}{2}
$$

Moreover, the temperature difference $\Delta T$ is given $\Delta T=T_{2}-T_{1} \quad$ if $T_{1}<T_{2}$

As a consequence, the dimensionless parameter $R_{T}$ can only take the values 0 or 1 . That is $R_{T}$ is 1 for asymmetric heating i.e. $T_{1}<T_{2}$, while $R_{T}$ is 0 for symmetric heating i.e. $T_{1}=T_{2}$, respectively. The dimensionless mean velocity $\bar{u}_{i}$ and the dimensionless bulk temperature $\theta_{b i}$ are given by

$$
\begin{aligned}
& \bar{u}_{1}=\int_{-1 / 4}^{0} u_{1} d y \\
& \bar{u}_{2}=\int_{0}^{1 / 4} u_{2} d y \\
& \theta_{b 1}=\frac{1}{\bar{u}_{1}} \int_{-1 / 4}^{0} u_{1} \theta_{1} d y \\
& \theta_{b 2}=\frac{1}{\bar{u}_{2}} \int_{0}^{1 / 4} u_{2} \theta_{2} d y
\end{aligned}
$$

Equation (8) implies that A can be either positive or negative. If $A>0$, then $U_{0}^{(i)}, \operatorname{Re}$ and $G R$ are negative, i.e. the flow is downward. On the contrary, if $A<0$, the flow is upward, so that $U_{0}^{(i)}$, Re and $G R$ are positive.

Using Eqs. (23) and (24), the Eqs. (13) to (22) become

Region-I

$\frac{d^{4} u_{1}}{d y^{4}}-M^{2} \frac{d^{2} u_{1}}{d y^{2}}=G R B r\left(\left(\frac{d u_{1}}{d y}\right)^{2}+M^{2} E^{2}+M^{2} u_{1}^{2}+2 M^{2} E u_{1}\right)$

Region-II

$\frac{d^{4} u_{2}}{d y^{4}}=m n K b h^{4} G R B r\left(\frac{d u_{2}}{d y}\right)^{2}$

The boundary and interface conditions become

$$
\begin{aligned}
& u_{1}=0 ; \quad \frac{d^{2} u_{1}}{d y^{2}}=-48+M^{2} E+\frac{G R R_{T}}{2} \\
& \text { at } y=-\frac{1}{4} \\
& u_{2}=0 ; \quad \frac{d^{2} u_{2}}{d y^{2}}=-48-\frac{n b G R R_{T}}{2} \\
& \text { at } y=\frac{1}{4} \\
& u_{1}=m h^{2} u_{2} ; \quad \frac{d u_{1}}{d y}=h \frac{d u_{2}}{d y} \quad \text { at } y=0 \\
& \frac{d^{2} u_{1}}{d y^{2}}-M^{2} u_{1}-M^{2} E=\frac{1}{n b}\left(\frac{d^{2} u_{2}}{d y^{2}}+48(1-n b)\right) \\
& \text { at } y=0 \\
& \frac{d^{3} u_{1}}{d y^{3}}-M^{2} \frac{d u_{1}}{d y}=\frac{1}{n b K h} \frac{d^{3} u_{2}}{d y^{3}} \quad \text { at } y=0
\end{aligned}
$$

\section{Solution}

\subsection{Case- I}

The solutions of Eqs. (30) and (31) using Eq. (32) in the absence of viscous dissipation, so that $B r=0$, the velocities become

\section{Region-I}

$u_{1}=B_{1}+B_{2} y+B_{3} \operatorname{Cosh}(M y)+B_{4} \operatorname{Sinh}(M y)$

Region-II

$u_{2}=B_{5}+B_{6} y+B_{7} y^{2}+B_{8} y^{3}$

Using the Eq. (23) in Eqs. (6) and (7), we obtain the energy balance equation as

Region-I 
J. Prathap Kumar et al. / JAFM, Vol. 5, No. 3, pp. 11-21, 2012.

$$
\theta_{1}=-\frac{1}{G R}\left(48+\frac{d^{2} u_{1}}{d y^{2}}-M^{2}\left(E+u_{1}\right)\right)
$$

Region-II

$$
\theta_{2}=-\frac{1}{n b G R}\left(48+\frac{d^{2} u_{2}}{d y^{2}}\right)
$$

Using the velocities obtained in Eqs. (33) and (34), the energy balance Eqs. (35) and (36) can be evaluated and are not presented.

\subsection{Case- II}

The solutions of Eqs. (30) and (31) can be obtained when buoyancy forces are negligible and viscous dissipation is dominating, i.e., $G R=0$, so that a purely forced convection occurs. For this condition solutions of Eqs. (30) and (31), using boundary and interface conditions given by Eq. (32) for $G R=0$, the velocities are given by

\section{Region-I}

$u_{1}=D_{1}+D_{2} y+D_{3} \operatorname{Cosh}(M y)+D_{4} \operatorname{Sinh}(M y)$

Region-II

$u_{2}=D_{5}+D_{6} y+D_{7} y^{2}+D_{8} y^{3}$

Using Eq. (23) in Eqs. (11) and (12), the energy balance equations become

Region-I

$\frac{d^{2} \theta_{1}}{d y^{2}}=-B r\left(\left(\frac{d u_{1}}{d y}\right)^{2}+M^{2} E^{2}+M^{2} u_{1}^{2}+2 M^{2} E u_{1}\right)$

Region-II

$\frac{d^{2} \theta_{2}}{d y^{2}}=-B r K m h^{4}\left(\frac{d u_{2}}{d y}\right)^{2}$

The boundary and interface conditions for temperature are

$\theta_{1}\left(-\frac{1}{4}\right)=-\frac{R_{T}}{2}$

$\theta_{2}\left(\frac{1}{4}\right)=\frac{R_{T}}{2}$

$\theta_{1}=\theta_{2} ; \frac{d \theta_{1}}{d y}=\frac{1}{K h} \frac{d \theta_{2}}{d y} \quad$ at $y=0$

Using Eq. (37), (38) and (41), the energy balance Eqs. (39) and (40) can be evaluated and not presented.

\subsection{Perturbation Solution}

We define the dimensionless parameter

$\varepsilon=\frac{G r}{\operatorname{Re}} B r=G R B r$

Equation (42) shows that $\varepsilon$ does not depend on the reference temperature difference $\Delta T$. The fact that the product $\operatorname{GR} \operatorname{Br}(=\varepsilon)$ is very small and hence can be exploited to use the regular perturbation method. To this end the solutions are assumed in the form:

$u_{i}(y)=u_{i 0}(y)+\varepsilon u_{i 1}(y)+\varepsilon^{2} u_{i 2}(y)+\cdots \cdots \cdots \cdots \cdot \cdots=\sum_{n=0}^{\infty} \varepsilon^{n} u_{i n}(y)$

Using Eq. (43) in Eqs. (30) and (31) and equating the coefficients of like powers $\varepsilon$ to zero, we obtain the zeroth and first-order equations as follows

Isothermal-isothermal $\left(T_{1}-T_{2}\right)$ wall conditions

Region-I

Zeroth-order equations:

$\frac{d^{4} u_{10}}{d y^{4}}-M^{2} \frac{d^{2} u_{10}}{d y^{2}}=0$

First-order equations:

$\frac{d^{4} u_{11}}{d y^{4}}-M^{2} \frac{d^{2} u_{11}}{d y^{2}}=\left(\frac{d u_{10}}{d y}\right)^{2}+M^{2} E^{2}+M^{2} u_{10}^{2}+2 M^{2} E u_{10}$

Region-II

Zeroth-order equations:

$\frac{d^{4} u_{20}}{d y^{4}}=0$

First-order equations:

$\frac{d^{4} u_{21}}{d y^{4}}=m n K b h^{4}\left(\frac{d u_{20}}{d y}\right)^{2}$

The corresponding boundary and interface conditions reduces to Zeroth-order equations:

$u_{10}=0 ; \frac{d^{2} u_{10}}{d y^{2}}=-48+M^{2} E+\frac{G R R_{T}}{2} \quad$ at $y=-\frac{1}{4}$
$u_{20}=0 ; \quad \frac{d^{2} u_{20}}{d y^{2}}=-48-\frac{n b G R R_{T}}{2}$

at $y=\frac{1}{4}$

$u_{10}=m h^{2} u_{20} ; \frac{d u_{10}}{d y}=h \frac{d u_{20}}{d y} \quad$ at $y=0$

$\frac{d^{2} u_{10}}{d y^{2}}-M^{2} u_{10}-M^{2} E=\frac{1}{n b}\left(\frac{d^{2} u_{20}}{d y^{2}}+48(1-n b)\right) \quad$ at $y=0$

$\frac{d^{3} u_{10}}{d y^{3}}-M^{2} \frac{d u_{10}}{d y}=\frac{1}{n b K h} \frac{d^{3} u_{20}}{d y^{3}} \quad$ at $y=0$

First-order equations:

$$
\begin{array}{ll}
u_{11}=0 ; \frac{d^{2} u_{11}}{d y^{2}}=0 & \text { at } y=-\frac{1}{4} \\
u_{21}=0 ; \frac{d^{2} u_{21}}{d y^{2}}=0 & \text { at } y=\frac{1}{4} \\
u_{11}=m h^{2} u_{21} ; \frac{d u_{11}}{d y}=h \frac{d u_{21}}{d y} & \text { at } y=0
\end{array}
$$




$$
\begin{aligned}
& \frac{d^{2} u_{11}}{d y^{2}}-M^{2} u_{11}=\frac{1}{n b} \frac{d^{2} u_{21}}{d y^{2}} \\
& \frac{d^{3} u_{11}}{d y^{3}}-M^{2} \frac{d u_{11}}{d y}=\frac{1}{n b K h} \frac{d^{3} u_{21}}{d y^{3}} \quad \text { at } y=0
\end{aligned}
$$

Solutions of zeroth-order Eqs. (44) and (46) using boundary and interface conditions (48) are

$$
\begin{aligned}
& u_{10}=C_{1}+C_{2} y+C_{3} \operatorname{Cosh}(M y)+C_{4} \operatorname{Sinh}(M y) \\
& u_{20}=C_{5}+C_{6} y+C_{7} y^{2}+C_{8} y^{3}
\end{aligned}
$$

Solutions of first-order Eqs. (45) and (47) using boundary and interface conditions (49) are

$$
\begin{aligned}
& u_{11}=C_{9}+C_{10} y+C_{11} \operatorname{Cosh}(M y)+C_{12} \operatorname{Sinh}(M y)+ \\
& g_{10} \operatorname{Cosh}(2 M y)+g_{11} \operatorname{Sinh}(2 M y)+g_{12} y^{2} \operatorname{Cosh}(M y) \\
& +g_{13} y^{2} \operatorname{Sinh}(M y)+g_{14} y \operatorname{Cosh}(M y) \\
& +g_{15} y \operatorname{Sinh}(M y)+g_{16} y^{4}+g_{17} y^{3}+g_{18} y^{2} \\
& u_{21}=g_{19} y^{8}+g_{20} y^{7}+g_{21} y^{6}+g_{22} y^{5}+g_{23} y^{4}+\frac{C_{13}}{6} y^{3}+\frac{C_{14}}{2} y^{2}+C_{15} y+C_{16}
\end{aligned}
$$

Using Eq. (23) in Eqs. (6) and (7), we obtain the energy balance equations as

Region-I

$\theta_{1}=-\frac{1}{G R}\left(48+\frac{d^{2} u_{1}}{d y^{2}}-M^{2}\left(E+u_{1}\right)\right)$

Region-II

$\theta_{2}=-\frac{1}{n b G R}\left(48+\frac{d^{2} u_{2}}{d y^{2}}\right)$

Using velocities obtained in Eqs. (50) to (53) the energy balance Eqs. (54) and (55) can be evaluated and are not presented.

\section{Isoflux-isothermal $\left(q_{1}-T_{2}\right)$ wall conditions}

For this case, the thermal boundary conditions for the channel walls can be written in the dimensional form as

$$
\begin{array}{ll}
q_{1}=-K_{1} \frac{d T_{1}}{d Y} & \text { at } Y=-\frac{h_{1}}{2} \\
T=T_{2} & \text { at } Y=\frac{h_{2}}{2}
\end{array}
$$

The dimensionless form of the above equations can be obtained by using the Eq. (23) along with $\Delta T=\frac{q_{1} h_{1}}{K_{1}}$ to give

$$
\begin{array}{ll}
\frac{d \theta_{1}}{d y}=-1 & \text { at } y=-\frac{1}{4} \\
\theta_{2}=\frac{R_{q t}}{2} & \text { at } y=\frac{1}{4}
\end{array}
$$

where $R_{q t}=\left(T_{2}-T_{0}\right) / \Delta T$ is the thermal ratio parameter for isoflux-isothermal walls. Other than the no-slip conditions at the channel walls, two more boundary conditions in terms of $U_{1}$ are required to solve Eq. (13). These are induced by the conditions given by Eq. (57) and the other obtained from Eq. (6) as follows.

Differentiating Eq. (6) with respect to $Y$ with $\frac{d P}{d X}=A$ gives

$\frac{d^{3} U_{1}}{d Y^{3}}-\frac{\sigma_{e} B_{0}^{2}}{\mu_{1}} \frac{d U_{1}}{d Y}+\frac{g \beta_{1}}{v_{1}} \frac{d T_{1}}{d Y}=0$

Equation (58) is non-dimensionalised by using the

Eq. (23) to give

$\frac{d^{3} u_{1}}{d y^{3}}-M^{2} \frac{d u_{1}}{d y}+G R \frac{d \theta_{1}}{d y}=0$

Evaluating the Eq. (59) at the left wall $(y=-1 / 4)$ yields

$\frac{d^{3} u_{1}}{d y^{3}}-M^{2} \frac{d u_{1}}{d y}=G R \quad$ at $y=-\frac{1}{4}$

The other boundary condition at the right wall can be shown to be the same as that given for the isothermalisothermal wall with $R_{T}$ is replaced by $R_{q t}$ such that

$\frac{d^{2} u_{2}}{d y^{2}}=-48-\frac{n b G R R_{q t}}{2} \quad$ at $y=\frac{1}{4}$

The integrating constants appeared in the Eqs. (50) to (53) are evaluated using boundary conditions (48), (49), (60) and (61) and are not presented

\section{Isothermal-isoflux $\left(T_{1}-q_{2}\right)$ wall conditions}

For this case, the thermal boundary conditions for the channel walls can be written in the dimensional form as
$q_{2}=-K_{2} \frac{d T_{2}}{d Y}$
at $Y=\frac{h_{2}}{2}$
$T=T_{1}$
at $Y=-\frac{h_{1}}{2}$

The dimensionless form of above equations can be obtained by using the Eq. (23) along with

$$
\Delta T=\frac{q_{2} h_{2}}{K_{2}}
$$

to give

$$
\begin{array}{ll}
\frac{d \theta_{2}}{d y}=-1 & \text { at } y=\frac{1}{4} \\
\theta_{1}=\frac{R_{t q}}{2} & \text { at } y=-\frac{1}{4}
\end{array}
$$

where $R_{t q}=\left(T_{1}-T_{0}\right) / \Delta T$ is the thermal ratio parameter for isothermal-isoflux walls. Other than the no-slip conditions at the channel walls, two more boundary conditions in terms of $U_{2}$ are required to solve Eq. (14). These are induced by the conditions given in Eq. (63) and the other obtained from Eq. (7) as follows. Differentiating Eq. (7) with respect to $Y$ with $\frac{d P}{d X}=A$ gives

$\frac{d^{3} U_{2}}{d Y^{3}}+\frac{g \beta_{2}}{v_{2}} \frac{d T_{2}}{d Y}=0$

Equation (64) is non-dimensionalised by using the Eq. (23) to give 
Table 1 Temperature values for different values of GR and $\varepsilon$.

\begin{tabular}{|c|c|c|c|c|c|c|}
\hline $\mathrm{y}$ & \multicolumn{3}{|c|}{$\mathrm{GR}=-500, \varepsilon=-0.1$} & \multicolumn{3}{c|}{$\mathrm{GR}=500, \varepsilon=0.1$} \\
\hline & $\mathrm{E}=-1$ & $\mathrm{E}=0$ & $\mathrm{E}=1$ & $\mathrm{E}=-1$ & $\mathrm{E}=0$ & $\mathrm{E}=1$ \\
\hline-0.25 & -0.5 & -0.5 & -0.5 & -0.5 & -0.5 & -0.5 \\
\hline-0.175 & -0.34976 & -0.34976 & -0.34975 & -0.34964 & -0.34964 & -0.34962 \\
\hline-0.1 & -0.19955 & -0.19955 & -0.19952 & -0.19954 & -0.19953 & -0.19949 \\
\hline-0.025 & -0.04945 & -0.04944 & -0.04941 & -0.04947 & -0.04946 & -0.04942 \\
\hline 0 & $5.56 \mathrm{E}-04$ & $5.61 \mathrm{E}-04$ & $5.89 \mathrm{E}-04$ & $5.44 \mathrm{E}-04$ & $5.50 \mathrm{E}-04$ & $5.78 \mathrm{E}-04$ \\
\hline 0.025 & 0.05054 & 0.05055 & 0.05057 & 0.05054 & 0.05054 & 0.05057 \\
\hline 0.1 & 0.20047 & 0.20047 & 0.20048 & 0.20044 & 0.20044 & 0.20045 \\
\hline 0.175 & 0.35037 & 0.35037 & 0.35037 & 0.35023 & 0.35023 & 0.35024 \\
\hline 0.25 & 0.5 & 0.5 & 0.5 & 0.5 & 0.5 & 0.5 \\
\hline
\end{tabular}

$\frac{d^{3} u_{2}}{d y^{3}}+G R n b \frac{d \theta_{2}}{d y}=0$

Evaluating the Eq. (65) at the right wall $(y=1 / 4)$ yields

$\frac{d^{3} u_{2}}{d y^{3}}=G R n b \quad$ at $y=\frac{1}{4}$

The other boundary condition at the right wall can be shown to be the same as that given for the isothermalisothermal wall with $R_{T}$ is replaced by $R_{t q}$ such that

$$
\frac{d^{2} u_{1}}{d y^{2}}=-48+\frac{G R R_{t q}}{2} \quad \text { at } y=-\frac{1}{4}
$$

The integrating constants appeared in the Eqs. (50) to (53) are evaluated using boundary conditions (48), (49), (66) and (67) and are not presented.

\subsection{Nuselt Number}

The heat transfer parameter on the wall expressed in terms of the Nuselt number, in non-dimensional form become

$$
\begin{array}{ll}
N u_{-}=(1+h) \frac{d \theta_{1}}{d y} & \text { at } y=-\frac{1}{4} \\
N u_{+}=\left(1+\frac{1}{h}\right) \frac{d \theta_{2}}{d y} & \text { at } y=\frac{1}{4}
\end{array}
$$

The expressions for $\mathrm{Nu} u_{-}$and $\mathrm{Nu} u_{+}$can be directly obtained and are not presented.

\section{RESUlts AND Discussion}

In this section the fluid flow and heat transfer results for an electrically conducting fluid flow in a vertical enclosure are discussed in the presence of an applied magnetic field $B_{0}$ normal to gravity and applied electric field $E_{0}$ parallel to gravity considering both viscous and homic dissipations. The electric loading parameter $E=0$ corresponds to short circuit configuration and $E \neq 0$ corresponds to open circuit, $E$ may be positive or negative depending either on the polarity of $E_{0}$ or on the sign of $T_{2}-T_{1}$. If the polarity of $E_{0}$ is fixed as positive, then $E$ may be positive or negative depending on $T_{2}>T_{1}$ or $T_{2}<T_{1}$ respectively. The effect of electromagnetic force when $E=0$ is found to accelerate the flow and hence acts as a MHD generator. Further, the direction of the flow when $E>0$ is opposite to that when $E<0$ and hence the present configuration can be used for effectively flow reversal situation required in many practical problems.

The basic equations governing the flow are solved using regular perturbation method. The product of $G R B r$, where $G R$ is the ratio of Grashof number to Reynolds number and $\mathrm{Br}$ is the Brinkmann number is used as the perturbation parameter.

The flow field for asymmetric heating are obtained and depicted in Figs. 2 to 10 and also shown in Tables 1 to 3 for the values of the parameters fixed as $m=1, b=1, h=1, K=1, n=1, M=2, G R=500$ and $\varepsilon=0.1$ expect the varying one. Equations (33) and (34) are the velocity field in both the regions in the absence of Brinkmann number and solutions are depicted graphically for $E=2, M=2$, in Fig. 2. There is a flow reversal near the cold wall at $y=-1 / 4$ for $G R=400$ and there is symmetric profile for $G R=0$.

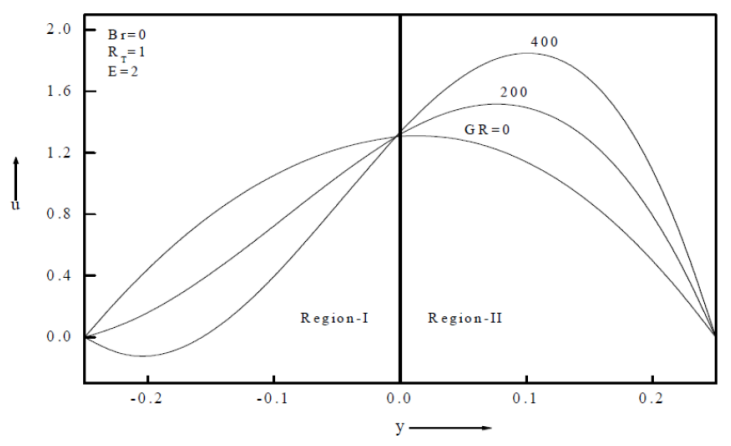

Fig. 2. Velocity profile s for different values of GR 
J. Prathap Kumar et al. / JAFM, Vol. 5, No. 3, pp. 11-21, 2012.

Table 2 Temperature profiles for different values of Hartmann number M.

\begin{tabular}{|c|c|c|c|c|c|c|}
\hline $\mathrm{y}$ & \multicolumn{3}{|c|}{$\mathrm{M}=2$} & \multicolumn{3}{c|}{$\mathrm{M}=6$} \\
\hline & $\mathrm{E}=-1$ & $\mathrm{E}=0$ & $\mathrm{E}=1$ & $\mathrm{E}=-1$ & $\mathrm{E}=0$ & $\mathrm{E}=1$ \\
\hline-0.25 & -0.5 & -0.5 & -0.5 & -0.5 & -0.5 & -0.5 \\
\hline-0.175 & -0.34976 & -0.34976 & -0.34975 & -0.34974 & -0.34977 & -0.3497 \\
\hline-0.1 & -0.19955 & -0.19955 & -0.19952 & -0.19954 & -0.19955 & -0.19944 \\
\hline-0.025 & -0.04945 & -0.04944 & -0.04941 & -0.04945 & -0.04943 & -0.04928 \\
\hline 0 & $5.56 \mathrm{E}-04$ & $5.61 \mathrm{E}-04$ & $5.89 \mathrm{E}-04$ & $5.48 \mathrm{E}-04$ & $5.77 \mathrm{E}-04$ & $7.25 \mathrm{E}-04$ \\
\hline 0.025 & 0.05054 & 0.05055 & 0.05057 & 0.05054 & 0.05056 & 0.0507 \\
\hline 0.1 & 0.20047 & 0.20047 & 0.20048 & 0.20047 & 0.20047 & 0.20055 \\
\hline 0.175 & 0.35037 & 0.35037 & 0.35037 & 0.35037 & 0.35035 & 0.35038 \\
\hline 0.25 & 0.5 & 0.5 & 0.5 & 0.5 & 0.5 & 0.5 \\
\hline
\end{tabular}

Table 3 Temperature profiles for different values of viscosity ratio $\mathrm{m}$.

\begin{tabular}{|c|c|c|c|c|c|c|}
\hline $\mathrm{y}$ & \multicolumn{3}{|c|}{$\mathrm{m}=0.1$} & \multicolumn{3}{c|}{$\mathrm{m}=4$} \\
\hline & $\mathrm{E}=-1$ & $\mathrm{E}=0$ & $\mathrm{E}=1$ & $\mathrm{E}=-1$ & $\mathrm{E}=0$ & $\mathrm{E}=1$ \\
\hline-0.25 & -0.5 & -0.5 & -0.5 & -0.5 & -0.5 & -0.5 \\
\hline-0.175 & -0.34992 & -0.34993 & -0.34993 & -0.34931 & -0.34931 & -0.3493 \\
\hline-0.1 & -0.19987 & -0.19989 & -0.19987 & -0.19868 & -0.19868 & -0.19865 \\
\hline-0.025 & -0.04986 & -0.04987 & -0.04986 & -0.04826 & -0.04824 & -0.04821 \\
\hline 0 & $1.35 \mathrm{E}-04$ & $1.25 \mathrm{E}-04$ & $1.39 \mathrm{E}-04$ & 0.00183 & 0.00185 & 0.00188 \\
\hline 0.025 & 0.05013 & 0.05012 & 0.05013 & 0.05186 & 0.05187 & 0.05191 \\
\hline 0.1 & 0.2001 & 0.2001 & 0.2001 & 0.20157 & 0.20158 & 0.2016 \\
\hline 0.175 & 0.35008 & 0.35007 & 0.35007 & 0.35112 & 0.35112 & 0.35113 \\
\hline 0.25 & 0.5 & 0.5 & 0.5 & 0.5 & 0.5 & 0.5 \\
\hline
\end{tabular}

Equations (44) and (45) are the solutions for temperature field in the absence of Grashof number which is depicted graphically in Fig. 3 for different values of Brinkmann number. The temperature field increases with increasing values of Brinkmann number. Fig. $2(B r=0)$ and $3(G R=0)$ are the similar graphs obtained by Umavathi (2006) and Baraletta (1998) for one fluid model considering permeable fluid and viscous fluid respectively.

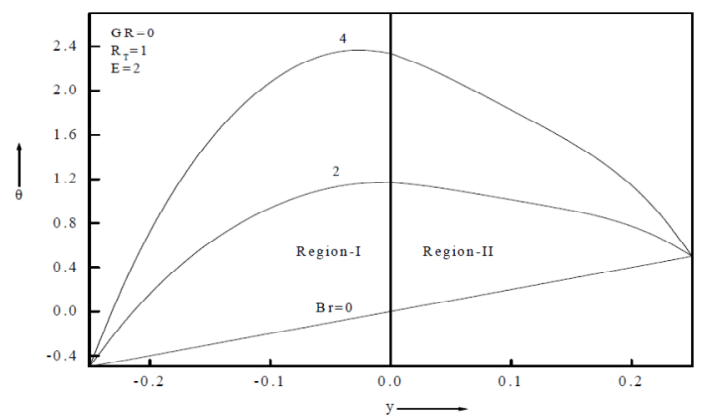

Fig. 3. Temperature profiles for different values of $\mathrm{Br}$.

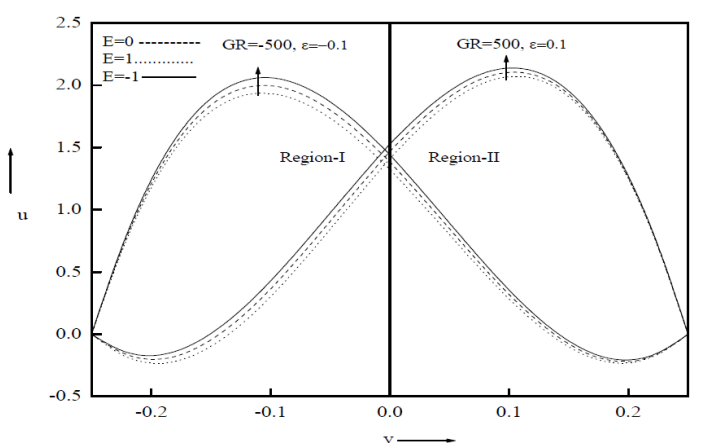

Fig. 4. Velocity profiles for different values of GR and $\varepsilon$.

The effect of $G R$ and $\varepsilon$ on the velocity is shown in Fig. 4 for short and open circuits. For positive values of $G R$ and $\varepsilon$ the flow reversal is near the cold wall and for negative values of $G R$ and $\varepsilon$ the flow reversal is near the hot wall for open and short circuits. The effect of $G R$ and $\varepsilon$ on temperature for $E=0, \pm 1$ is shown in Table 1 . 
Table 4 Nusselt values for different values of Hartmann number M.

\begin{tabular}{|c|c|c|c|c|c|c|}
\hline \multirow{2}{*}{$\mathrm{M}$} & \multicolumn{2}{|c|}{$\mathrm{E}=-1$} & \multicolumn{2}{c|}{$\mathrm{E}=0$} & \multicolumn{2}{c|}{$\mathrm{E}=1$} \\
\cline { 2 - 7 } & $\mathrm{Nu}-$ & $\mathrm{Nu}+$ & $\mathrm{Nu}-$ & $\mathrm{Nu}+$ & $\mathrm{Nu}-$ & $\mathrm{Nu}+$ \\
\hline 2 & 14.42359639 & 3.979748009 & 14.42348712 & 3.980019086 & 14.42395032 & 3.980101942 \\
\hline 4 & 4.658277671 & 3.979524633 & 4.657859532 & 3.980437548 & 4.659464179 & 3.980711141 \\
\hline 6 & 4.136257668 & 3.979341515 & 4.135371929 & 3.980957939 & 4.13834317 & 3.981427017 \\
\hline
\end{tabular}

The variation of temperature for positive and negative $G R$ and $\varepsilon$, the values are same up to three decimal places and effect is almost invariant for both open and short circuits.

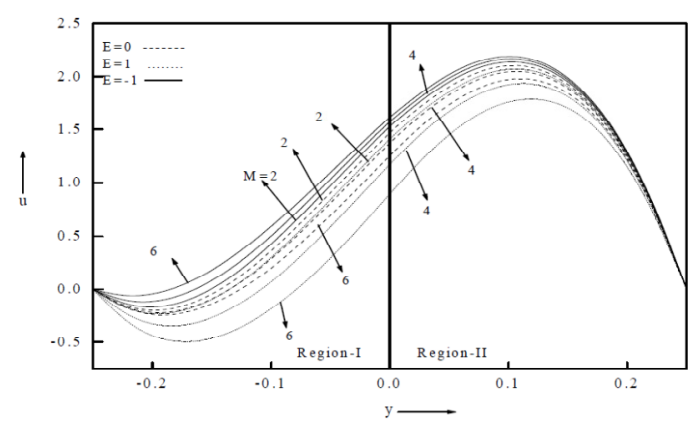

Fig. 5. Velocity profiles for different values of Hartmann number $\mathrm{M}$.

The effect of Hartmann number on velocity is shown in Fig. 5. It is observed that the Hartmann number is to decrease the velocity in both the regions. This graph also shows the effect of electric field load parameter $E$ on the flow for both open and short circuits. Effect of Hartmann number is to suppress the velocity. The effect of Hartmann number on temperature varies from fifth decimal place as shown in Table 2 for both open and short circuits.

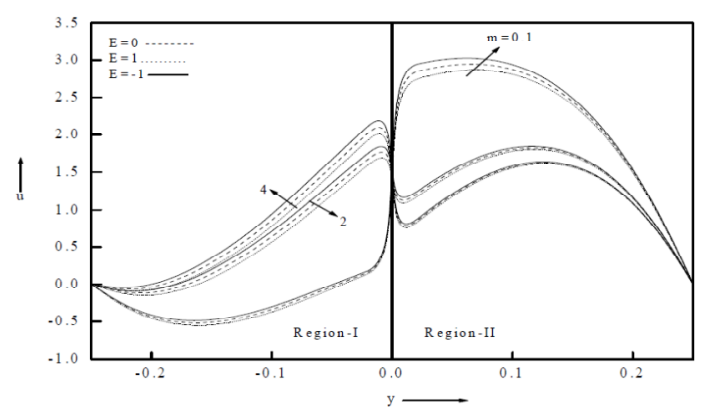

Fig. 6. Velocity profiles for different values of viscosity ratio $\mathrm{m}$.

Figure 6 shows the effect of viscosity ratio $m$ on the velocity. It is found that larger the value of viscosity of conducting fluid in region-I compared to the viscosity of another conducting fluid in region-II, the larger the flow field in region-I and smaller the flow field in region-II for both open and short circuits. For $m<1$, flow reversal is observed near the cold wall. The effect of viscosity ratio $m$ on the temperature is very sensitive and it varies from fourth decimal place as shown in Table 3 for $E=0, \pm 1$.
The effect of width ration $h$ on velocity and temperature are shown in Figs. 7 and 8 respectively. As the width ratio $h$ increases velocity decreases significantly in region-II when compared to region-I. It is found that smaller the width of region-I compared to region-II, smaller the temperature.

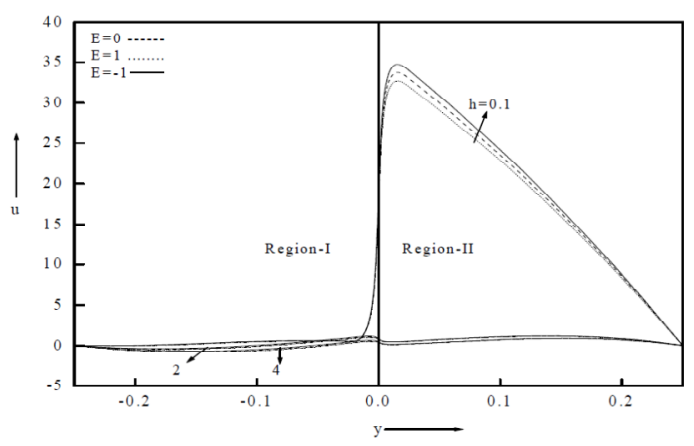

Fig. 7. Velocity profiles for different values of width ratio $h$.

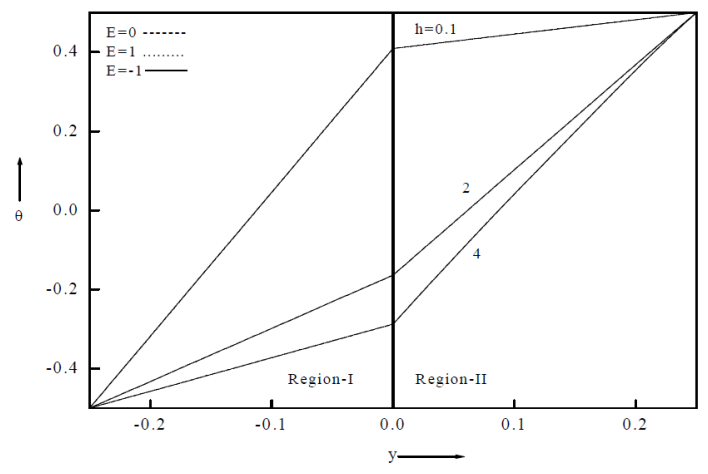

Fig. 8. Temperature profiles for different values of width ratio $h$.

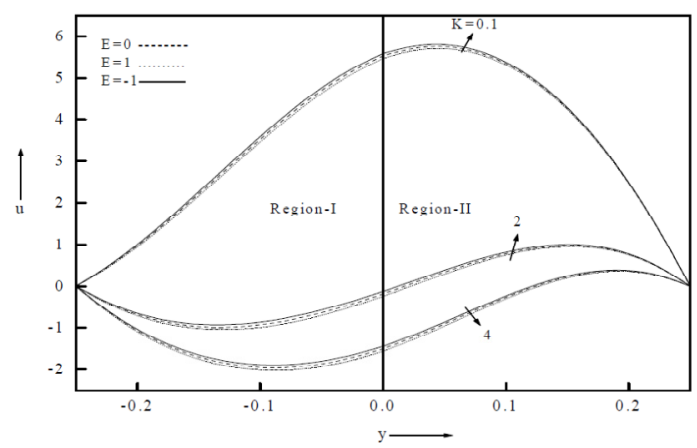

Fig. 9. Velocity profiles for different values of thermal conductivity ratio $\mathrm{K}$. 


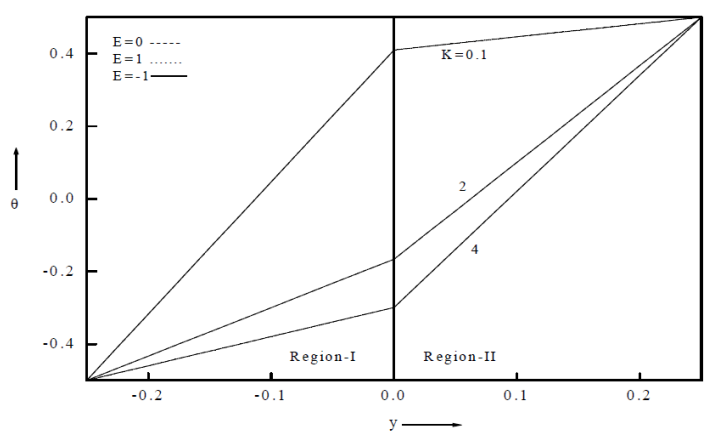

Fig. 10. Temperature profiles for different values of thermal conductivity ratio $\mathrm{K}$.

It is interesting to note that the velocity and temperature are invariant for $E=0, \pm 1$ for varying width ratio $h$.

The effect of thermal conductivity ratio $K$ on the flow is shown in Figs. 9 and 10. It is seen that increasing the value of $K$ decreases both velocity and temperature in both the regions. There is a flow reversal near the cold wall. There is no effect of electric field load parameter $E$ on the flow for varying $K$.

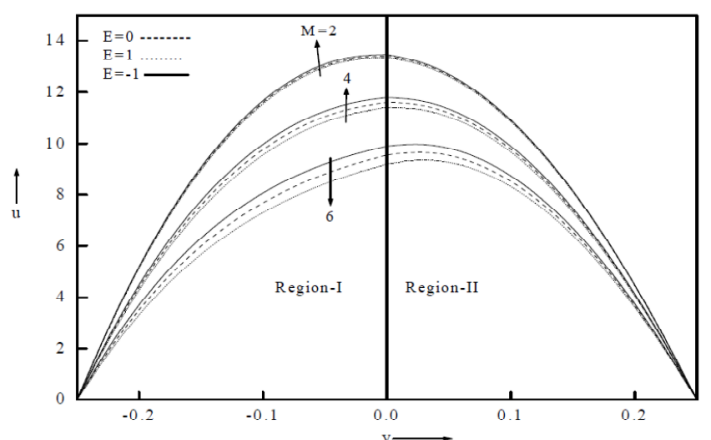

Fig. 11. Velocity profiles for different values of Hartmann number $\mathrm{H}$ in isoflux-isothermal wall conditions.

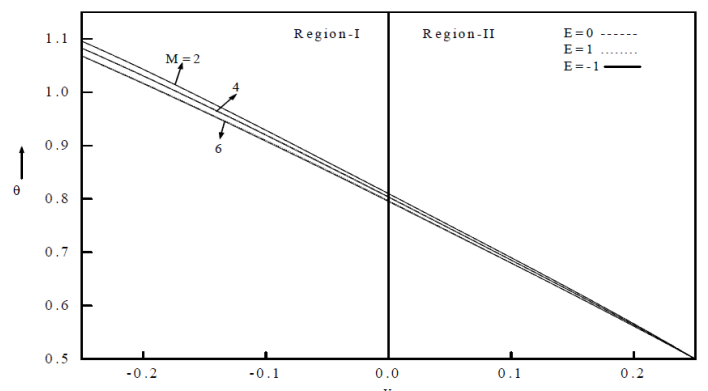

Fig. 12. Temperature profiles for different values of Hartmann number $\mathrm{M}$ in isoflux-isothermal wall conditions.

Figures 11 to 14 shows the effect of Hartmann number $M$ on the flow for isoflux-isothermal and isothermalisoflux wall conditions for $E=0, \pm 1$. The effect of Hartmann number $M$ is to suppress the velocity and temperature in both the regions as seen in Figs. 11 and 12 respectively. The velocity is suppressed for both open and short circuits where as the effect of electric load parameter $E$ on temperature is invariant.
For isothermal-isoflux wall conditions, the effect of Hartmann number is to promote the velocity and temperature in both the regions but the direction of flow is reverse when compared to isoflux-isothermal wall conditions. In this case also the effect of electric field parameter $E$ is significant on velocity and invariant on temperature.

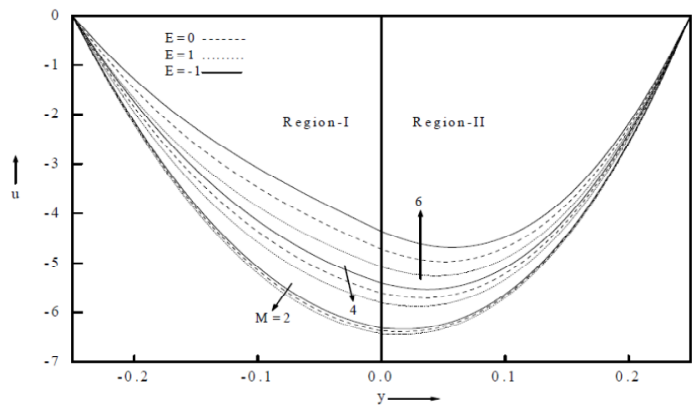

Fig. 13. Velocity profiles for different values of Hartmann number $\mathrm{M}$ in isothermal-isoflux wall conditions.

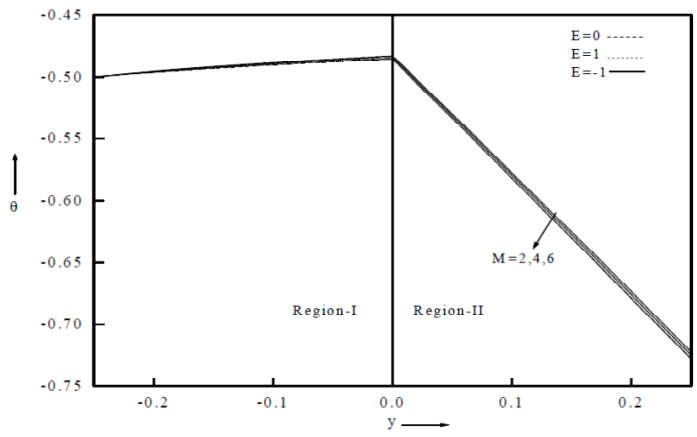

Fig. 14. Temperature profiles for different values of Hartmann number $\mathrm{M}$ in isothermal-isoflux wall conditions.

The variations of Nusselt number for different values of Hartmann number for both open and short circuits is shown in Table 4. For $E=-1$, as the Hartmann number $M$ increases, rate of heat transfer decreases near the cold wall and near the hot wall but magnitude is very large near the cold wall. Similar result is observed for short circuit $(E=0)$ and for open circuit $(E=1)$ on Nusselt number for varying Hartmann number. .

\section{REFERENCES}

Aung, W. (1987). Mixed convection in Internal flow, in: S. Kakac, R.K.Shah, W. Aung (Eds.), Handbook of Single-phase Convective Heat Transfer. Wiley, New York (Chapter 15).

Aung, W., and G. Worku (1987). Mixed convection in ducts with asymmetric wall heat Fluxes. ASME J. Heat Transfer 109, 947-951.

Aung, W., and G. Worku (1986a). Theory of fully developed combined convection including flow reversal. ASME J. Heat Transfer 108, 485-488.

Aung, W., and G. Worku (1986b). Developing flow and flow reversal in a vertical channel with 
asymmetric wall temperature. ASME J. Heat Transfer 108, 299-304.

Bentwich, M. (1964). Two-phase axial flow in pipe. Basic Eng.D. 86, 669-672.

Bird, R.B., W.E. Stewart and E.N. Lightfoot (1960). Transport Phenomena. Wiley, New York.

Charles, M.E. and L.U. Lilleleht (1965). Co-current stratified laminar flow of two immiscible liquids in a rectangular conduit. Can.J.Chem. Engng. 43, 110-116.

Cheng, C.H., H.S. Kou and W.H. Huang (1990). Flow reversal and heat transfer of fully developed mixed convection in vertical channels $J$. Thermophys. Heat Transfer 4, 375-383.

Dhir, V.K. (1997). Heat Transfer from heat-generating pools and particulate beds. Adv. Heat Transfer 29, $1-57$.

Fieg, G. (1976). Experimental investigation of heat transfer characteristics in liquid layers with internal heat sources, in: Proc. Int. Meet. On Fast Reactor Safety and Related Physics, USERDA Conf 761001, 2047-2055.

Hamadah, T.T. and R.A. Wirtz (1991). Analysis of laminar fully developed mixed convection in a vertical channel with opposing buoyancy. ASME J. Heat Transfer 113, 507-510.

Ingham, D.B., D.J. Keen and P.J. Heggs (1988). Flows in vertical channels with asymmetric wall temperatures and including situations where reverse flows occur. ASME J. Heat Transfer 110, 910-917.

Kulacki, F.A. and A.T. Nguen (1981). Hydrodynamic instability and thermal convection in a horizontal layer of two immiscible fluids with internal heat generation. NUREG/CR-2619 Report.

Malashetty, M.S., J.C. Umavathi and J. Prathap Kumar (2006). Magnetoconvection of two-immiscible fluids in vertical enclosure. Heat Mass Transfer 42, 977-993.

Malashetty, M.S., J.C. Umavathi and J. Prathap Kumar (2001). Convectivemagneto hydrodynamic two fluid flow and heat transfer in an inclined channel.Heat and Mass transfer 37, 259-264.

Malashetty, M.S., J.C. Umavathi and J. Prathap Kumar (2000). Two fluid Magnetoconvecion flow in an inclined channel. Int. J. Transport phenomena. $3,73-84$.

Malashetty, M.S. and J.C., Umavathi (1997). TwoPhase Magnetohydrodynamic Flow and Heat Transfer in an Inclined Channel. Int. J. Multiphase Flow 23, 545-560.
Malashetty, M.S. and V. Leela (1992). Magnetohydrodynamic Heat Transfer in Two Phase Flow. Int. J. Eng. Sci., 30, 371-377.

Malashetty, M.S. and V. Leela (1991). Magnetohydrodynamic Heat Transfer in TwoFuid Flow.- Proceeding of the National Heat Transfer, Conf., AIChE and ASME, HTD, 159.

Mitra, P. (1982). Unsteady flow of two electrically conducting fluids between two rigid parallel plates. Bulletin of the Calcutta Mathematical Society 74, 87-95.

Pacham, B.A. and R. Shail (1971). Stratified laminar flow of two immiscible fluidsCamb. Phil. Soc. 69, 443-448

Schramm, R. and H.H. Reineke (1978). Natural convection in a horizontal layer of two different fluids with internal heat sources, in: Proc. $6^{\text {th }}$ Int. Heat Transfer Conf., National Research Council of Canada Paper NC-20.

Sehgal, B.R. (2001). Accomplishments and challenges of severe accident research. Nucl. Eng. Des. 201, 79-94.

Shail, R. (1973). On laminar two-phase flows in magnetohydrodynamics. Int. J. Engng. Sci. 11, 1103-1108.

Tao, T.N. (1960). On combined free and forced convection in channels. ASME J. Heat Transfer, $82,233-238$ 